D INSTITUTE FOR DEFENSE ANALYSES

NSD-5174

Agility Quotient (AQ)

David S. Alberts

April 2014

Institute for Defense Analyses

4850 Mark Center Drive

Alexandria, Virginia 22311-1882

Approved for public release; distribution is unlimited.

IDA Log No. H 14-000486 
The Institute for Defense Analyses is a non-profit corporation that operates three federally funded research and development centers to provide objective analyses of national security issues, particularly those requiring scientific and technical expertise, and conduct related research on other national challenges.

About This Publication

This work was conducted by the Institute for Defense Analyses (IDA) under contract DASW01-04-C-0003, Task AA-6-3630, "DoD Command and Control Research Program (DoD CCRP) Support," performed for the Command and Control (C2) Programs and Policy Directorate, Office of the Under Secretary of Defense for Acquisition, Technology \& Logistics, (AT\&L). The views, opinions, and findings should not be construed as representing the official position of either the Department of Defense or the sponsoring organization. 
NSD-5174

Agility Quotient (AQ)

David S. Alberts

April 2014 



\section{$19^{\text {th }}$ ICCRTS}

“C2 Agility: Lessons Learned from Research and Operations”

Track 1 Paper 072

Agility Quotient (AQ)

David S. Alberts

Institute for Defense Analyses

4850 Mark Center Drive

Alexandria, VA 22311

USA

$+1-703-845-2411$

dalberts@ida.org 



\title{
Agility Quotient (AQ)
}

\author{
David S. Alberts \\ Institute for Defense Analyses
}

\begin{abstract}
Agility, the capability to successfully effect, cope with, and/or exploit changes in circumstances, can be directly observed only when this capability has been manifested. Increasingly agility is seen as an essential system capability and hence a requirement. To satisfy a stated requirement for agility we need to be able to answer two questions. "How can we measure a system's Agility IQ?" and "What is the requisite amount of Agility that is required?" This paper suggests a way forward and illustrates it, in the context of C2 systems.
\end{abstract}

\section{Introduction}

Agility is increasingly being recognized as a 'must-have' capability for individuals, organizations, and the systems that support them as enterprises are called upon to successfully perform, if not thrive, in environments that are increasingly complex and dynamic. Commanders and managers at all levels are faced not only with the challenge of assuring that their organizations perform under these challenging conditions, but must accomplish this in an increasingly constrained budget environment that limits the resources that are available to hedge one's bets. Thus, efficiency must be considered along with effectiveness and risk.

The concept of Agility and its six enablers, as articulated in a number of U.S. Department of Defense Command and Control Research Program (DoD CCRP) publications, provides a conceptual framework that can be employed to find an appropriate balance between and among effectiveness, efficiency, and risk. This conceptual framework provides a rational basis for improving an entity's agility ${ }^{1}$. Progress in designing and developing more agile entities will depend on our ability to observe appropriate behaviors and outcomes, associate them with entity characteristics, and determine the amount of agility required. This paper focuses on a critical enterprise function, command and control. It discusses the need to complement agility assessments based upon observation of manifest agility with an a scenario free assessment approach that provides a measure of agility potential, an "Agility Quotient (AQ).”

While the development of a model of potential agility is outside its scope, this paper identifies a number of questions that could lead to the identification of key variables and relationships that should be included in a model of Command and Control Agility Potential whose output would be an entity's C2 AQ.

\footnotetext{
${ }^{1}$ Entity is used here to refer to the 'unit' of analysis whether it be an individual, a group of individuals, a formal organization, a coalition, a process, a policy, or a system.
} 


\section{Defining Agility}

To paraphrase some common wisdom, the only thing certain about the future is uncertainly. Furthermore, the situations we face are and will be dynamic. Therefore, solutions will become less effective over time and will need to be re-invented. Plans will be, at best, short-lived. This is because no matter how much we invest in information and uncertainly reduction, a significant amount of residual uncertainty will remain. As a result, we will always need to deal with unexpected and unanticipated events and circumstances. Agility is the only other way to meet the challenges of complexity and dynamics because Agility, defined as the "capability to successfully effect, cope with, and exploit changes in circumstances"” is not about "reducing problem difficult but is a way of dealing with complexity and uncertainty." 3 While this definition of Agility is widely used within the $\mathrm{C} 2$ Research community, different communities define "Agility" in different ways and/or employ a variety of terms (e.g., robustness, resilience, reliability) for this capability. However, despite their differences, different definitions of agility converge on three key ideas ${ }^{4}$.

The first key idea is that agility is an appropriate response to the challenges posed by complexity and dynamics. Increased system complexity and dynamics are associated with a reduced ability to predict. Consequently, these challenges are characterized by a rise in the frequency of unanticipated events. Increased complexity is also associated with exacerbating the adverse consequences of these events particularly as they may trigger cascades of effects that cannot be adequately understood or controlled. This leads to an increased probability of catastrophic failure.

The second key idea is that agility is inseparable from success, that is, an appropriate measure of agility must reflect outcomes. Thus, an entity manifests agility only if and when it: 1) can seize upon an opportunity to improve performance, increase efficiency and/or reduce risk; or, 2) is able to continue to operate successfully despite being put under a stress that would otherwise adversely impact its ability to operate successfully.

The third key idea is that agility is not a passive concept, but one that includes anticipatory and proactive behaviors in addition to being able to respond to challenges.

\section{Observing and Measuring Agility}

Agility is defined as a capability that results in a specific outcome under specific conditions. The specified outcome is success, where success is defined either by the entity itself or external to the entity. Depending on the nature of the entity, success will be determined by some combination of performance or effectiveness, costs, and risks. The condition specified is that of change, either in the mission (task), the environment, or in the circumstances to include the state of the entity.

\footnotetext{
${ }^{2}$ NATO SAS-085 C2 Agility Report (2013) p. 20 http://dodccrp.org/sas-085/sas-085 report final.pdf

${ }^{3}$ Alberts, David S., The Agility Advance (2011) DoD CCRP p.61 http://dodccrp.org/files/agility advantage/Agility Advantage Book.pdf

${ }^{4}$ For a fuller discussion, see Dove and LaBarge (2014) Fundamentals of Agile Systems Engineering
} 
While success or a lack thereof can usually be easily observed, the reasons are often less apparent. Clearly one can conceive of numerous reasons why an entity might be successful in spite of itself or its capabilities, to include Agility. This would suggest that an observation of success alone should not be equated with Agility. First, there needs to be a significant change that has occurred, one that can change the outcome. Thus, for an entity to be said to manifest Agility, the entity: 1) needs to be agile; 2) needs to maintain acceptable performance or improve performance; and, 3 ) possess some agility-related capability that could account for the outcome.

The following six agility-related capabilities or the enablers of agility have been identified:

- Responsiveness

- Versatility

- Flexibility

- Resilience

- Adaptiveness

- Innovativeness

Although it is difficult, if not impossible, in real world situations to establish a cause effect relationship between a successful outcome and the presence of these enablers, it is possible to observe these enablers (or a lack thereof) in entity behaviors and employ a measure of the degree to which the enabler is present. Thus, manifest agility and its impacts can be directly observed and measured, but only when circumstances that require agility occur, only if the entity in question is able to, as least in part, appropriately responds to the challenge at hand.

When an entity does not possess adequate agility, this lack of agility can also be observed. A metric used to measure entity agility can be calculated from these observations. It can be quite simple. For example, one metric is the probability that an entity manifests agility when required. Calculating this probability is a three-step process. The first step involves the construction of an Endeavor Space, a space that includes the population of missions and circumstances. This provides the set of missions and circumstances that need to be analyzed to determine if the entity can successfully operate in different parts of the Endeavor Space. The second step is to project whether or not the entity can successfully operate in each part of the space. ${ }^{5}$ The third step is to sum the outcomes across the Endeavor Space. This provides an overall probability of success, a measure of potential agility.

Success and failure have costs associated with them. One way to determine the value of agility is to take into consideration the amount of time an entity could not acceptably perform and the magnitude of the performance shortfall as is depicted in Figure 1.

\footnotetext{
${ }^{5}$ A more sophisticated analysis could estimate the conditional probability of success (see Alberts (2011): The Agility Advantage DoD CCRP - section on Impact of Loss of Connectivity, p. 394-8 ) http://dodccrp.org/files/agility advantage/Agility Advantage Book.pdf
} 


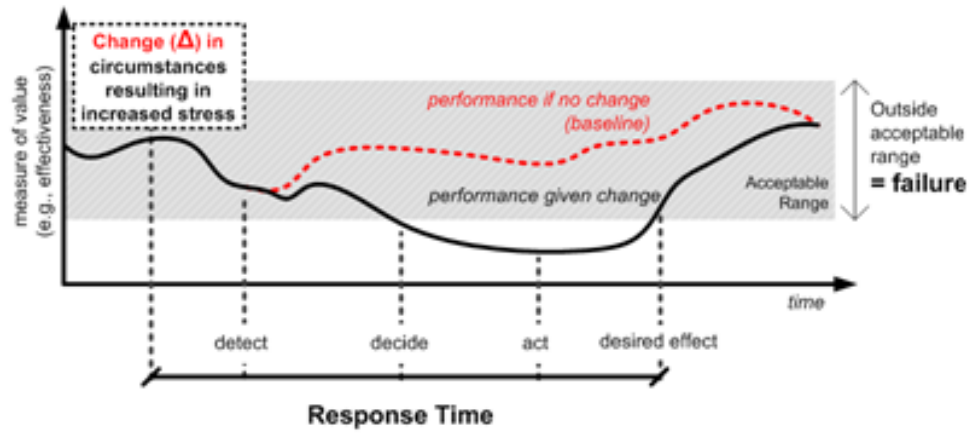

Figure 1: Observing Entity Performance

Figure 1: Observing Entity Performance is a graph that provides some very useful information about entity performance under stress or in the event of changed circumstances. Specifically, Figure 1 provides an anatomy of a response time-line that, if improved, could reduce the amount of time the system failed to perform within acceptable bounds and/or decrease the amount of the performance gap. Figure 1 shows us what the impact to performance was but not why. This limits our ability to understand what there is about the entity itself (its design and characteristics, its behaviors, and the performance of supporting systems) that may be contributing to this result. We cannot, without other information, forensically determine if or which of the enablers or inhibitors of agility came into play. If other observations were made, as was the case in the SAS-085 case studies, ${ }^{6}$ the presence or absence of these enablers could be determined and, in cases when they were presence, a successful outcome could be considered to be a manifestation of agility.

Being able to observe entity behavior and to note instances of manifest agility (or a lack thereof) and measure the impacts, does not, in of itself, allow us to adequately assess entity agility. This is because if we were to limit our assessments of an entity's agility to those instances when there is a requirement for agility, our measure of entity agility would be biased.

While measuring manifest agility by looking at the proportion of times an entity successfully performed under adversity can, if an entity receives low marks, provide systems engineers and operators with an indication of a problem and thus be helpful. High marks should not be considered to be proof of adequate agility. This is because the sample of stresses and conditions considered is both extremely small and not necessarily representative of future conditions. This

\footnotetext{
${ }^{6}$ SAS-085 Case Study methodology and results can be found in Chapter 7 of the Final Report and in Appendix B http://dodccrp.org/html4/sas-085.html
} 
is, of course, a gross understatement. The very reason we need increased agility is that past performance is a poor predictor of future performance. History and experience amply confirm this.

Clearly we need not limit our assessment of agility by only using observations of an entity in operation. One approach that is used to augment real world observations and thus improve our ability to predict performance is to put the system or a simulation of the system in a controllable, instrumented environment and create possible futures. Doing so allows us to test the system under a variety of scenarios without waiting for them to occur in the real world. The set of scenarios that is used, plus those scenarios that are thought to be 'lesser included cases,' constitute the design or Endeavor Space. Appropriated formulated, the Endeavor Space can be used to calculate either an absolute measure of agility, a probability of success, or a relative measure that simply compares two system designs or instantiations to the same standard. System engineering practice is focused upon creating a design space that corresponds to "requisite” agility.

Designing and testing an entity to these standards is thought to provide a better estimate of future behavior (success) than simply using past performance. However, it could be argued that the scenario-based approach could actually result in a less accurate prediction. Whether using a set of selected scenarios provides a more accurate assessment of system agility, depends upon the number and nature of the scenarios utilized, that is, the completeness of the Endeavor Space. The reason why a scenario-based approach is problematic has to do with the difficulty of developing a representative set of scenarios, one that adequately covers future situations. Some designers tend to focus on the 'most likely' situations and stresses while others focus on the most stressing circumstances they can think of. In either case, it seems inevitable that the set of scenarios employed will be constrained by pre-conceived notions, group think, and biases. Empirical evidence supports this observation.

The take away is not that we should abandon a scenario-based approach, but rather that we should be careful to employ scenarios in a thoughtful way. Given the limitations of the scenario approach, it seems prudent that we should also employ an alternate approach to estimating an entity’s potential agility.

The Agility Quotient “AQ”: A Measure of Potential

Given the limitations of measures of manifest agility, the development of a measure of an entity's AQ or potential agility is worth our attention. AQ can be patterned after the Intelligence Quotient (IQ). IQ is a score that is associated with educational potential, an ability to learn. That is, a high IQ score is considered to be a predictor of education success while a low score with a lack of success. Thus, AQ, as envisioned in this paper, seeks to be a predictor of success in the face of complexity and dynamics.

The IQ tests with which we are familiar seek to measure cognitive capabilities such as attention, memory, and problem solving ability. In other words, they are attempts to measure fundamental attributes or capabilities of individuals that enable them to comprehend, acquire knowledge, and apply it in useful ways. Since their inception in the early 1900s, the developers of these tests 
recognized that intelligence is too encompassing a concept to be measured in a scalar metric ${ }^{7}$. Furthermore, there were a host of factors besides genetics that could influence intelligence and along with other factors could bias test results. We need to keep this in mind as we try to develop AQ tests and explore ways to express the results of these tests quantitatively.

As we develop ways of determining and measuring an entity's AQ, it seems reasonable to initially build upon the enablers of agility that have previously been identified and to some extent validated in case studies and experiments. ${ }^{8}$ Figure 2: Enablers of Agility ${ }^{9}$, depicts the six enablers of agility identified above in the context of the characteristics that make tasks difficult and/or conditions that stress an entity. Responsiveness is required to accommodate time pressures. Resilience is required to recover from damage or degradation. Flexibility is needed when one way of accomplishing something does not work. Versatility is needed when an entity is used for multiple purposes. Innovativeness is required when existing ways and means are not adequate for the task and circumstances. Adaptiveness is required when, to succeed, the entity needs to change itself. The ability of an entity to change itself includes but is not limited to being able to adopt different approaches to C2 (see A Model of Potential C2 Agility ${ }^{10}$ ).

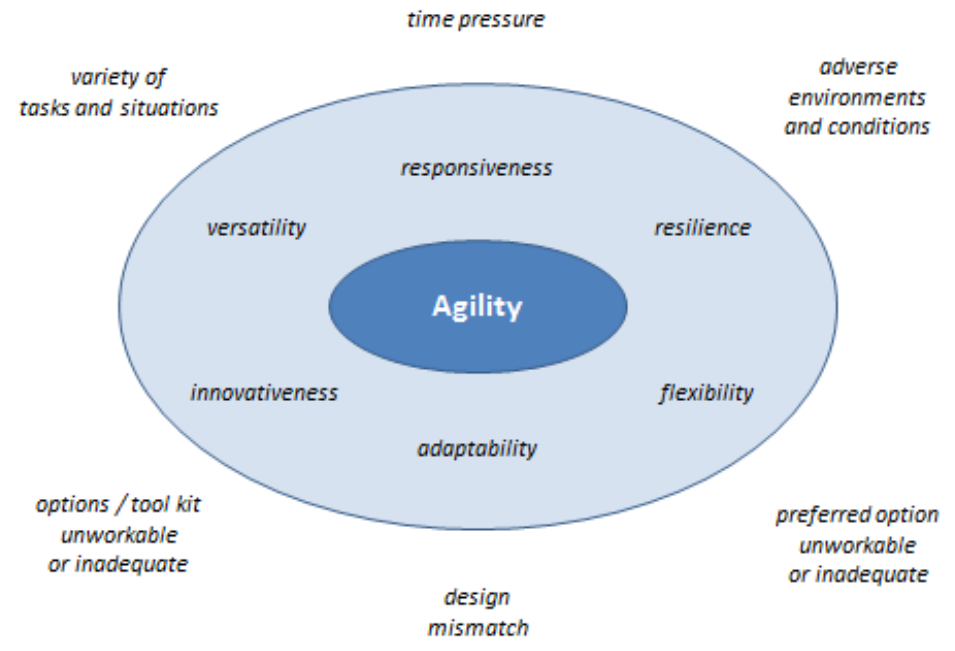

Figure 2: Enablers of Agility

The relationship between the presence (or absence) of these enablers and an entity's ability to remain at an acceptable level of performance (or improve on its performance) is nonlinear and could reasonably be expected to follow the form of a traditional stimulus-response curve for

\footnotetext{
${ }^{7}$ See Kendra Cherry, History of Intelligence Testing, http://psychology.about.com/od/psychologicaltesting/a/int-history.htm

${ }^{8}$ http://dodccrp.org/sas-085/sas-085 report final.pdf

${ }^{9}$ Adapted from Alberts, D.S. and Hayes, R.E, Understanding Command and Control http://www.dodccrp.org/files/Alberts UC2.pdf

${ }^{10}$ Alberts (2011) Part VI Potential Agility pp 451-516
} 
each enabler. For example, there is a certain threshold amount of resilience before it has any impact on outcomes and there is a limit to how much flexibility can be employed productively. These relationships are further complicated by the fact that the enablers are not mutually independent. For example, responsiveness can be enhanced by resilience. As is the case with agility itself, these enablers do not provide boundless value.

Since there are costs associated with designing and building agility enablers into an entity, tradeoffs need to be made between and among investments in these enablers. These tradeoffs can be informed by a model of potential agility (variables that represent each of these enablers, the relationships between and among them, and with entity agility). The output of this model would be a measure of Entity AQ, one that is independent of specific scenarios.

\section{Model of an Entity’s Potential Agility (AQ Test)}

A model of an entity's potential agility seeks to explain why an entity manifests agility when it does and also why it fails to exhibit agile behaviors. The development of such a model is informed by an understanding of how an entity behaves under stress and the processes by which an entity is changed to adapt and evolve. As such, a model of potential agility is not static but will be refined over time as our understanding increases.

Entities differ widely with respect to their purposes, the environments in which they operate, and in the nature of the components from which they are assembled, the specific set of variables that must be included in a model of potential agility will differ as well. Furthermore, even for entities that may have similar challenges and characteristics, the values of the parameters associated with the relationships between and among the variables that form a model of potential agility will differ. Despite these differences, system (domain) specific models should be derived from a generic model of potential agility based upon the enablers of agility and the relationships between and among these enablers. This will help: facilitate their development; ensure that all aspects of agility are considered in a systematic fashion; and, facilitate cross-pollination that can serve to accelerate the model maturation. Hence, domain-specific models of an entity's potential agility are instantiations of a generic model.

Over time, entities (individuals, organizations, and systems) have evolved and as a result have learned (adapted) so that they excel in dealing with a set of known and expected stresses. The means they have found useful, along with the enablers of agility, constitute a starting point for the construction of an entity specific model of potential agility. In the remainder of this paper, two examples are provided to illustrate how one can develop a model of potential agility that can be used to form the basis of an AQ test.

\section{A Model of C2 Agility Potential (C2 AQ)}

There are many ways to accomplish the functions we associate with C2. These different ways or C2 Approaches, correspond to different regions in the C2 Approach Space. The three dimension C2 Approach Space is depicted in Figure 3. These dimensions are not independent 
as how decision rights are allocated will impact the patterns of interactions and both of these will in large part determine how information is distributed.

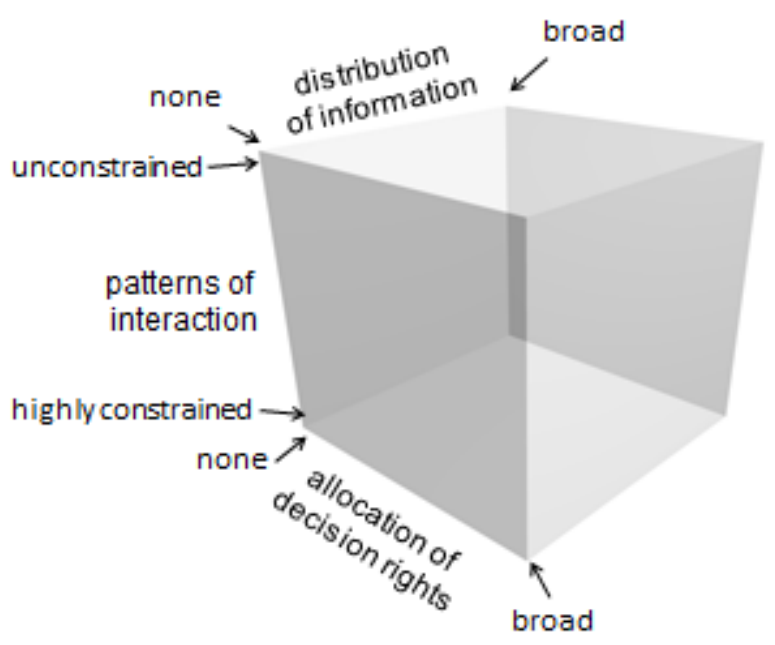

Figure 3: C2 Approach Space

Experience, case studies, and experiments have yielded many C2 Agility-related findings. These findings form the basis for the following set of hypotheses that bear directly on the development of a model of C2 AQ:

- there is no 'one size fits all' approach to C2 that works well for all missions and circumstances;

- Network-enabled approaches to C2 are more agile then others;

- Balanced approaches to C2 are more agile;

- The 'selected' approach to C2 may not be the one that is actually being implemented;

- $\quad$ being able to appropriately adopt more than one approach improves agility (C2 Maneuver); and,

- agile individuals, processes, policies, and systems each, and in combination, improve both the agility of a given C2 approach and the ability to appropriately maneuver in the C2 Approach Space.

To the extent that these hypotheses have merit, an entity's C2 Agility is a function of the: 1) number of different C2 Approaches that it can adopt; 2) agility of each of these C2 Approaches; and, 3) ability to appropriately maneuver in the C2 Approach Space. A model of Potential C2 Agility needs to include these contributors to agility, the relationships 
between and among them, and the factors that impact the extent to which they contribute to agility.

The C2 Agility-related lessons learned highlighted above suggest a number of questions, the answers to which would provide some indication as to an entity's C2 AQ and point to some observables that could be used to construct a model of C2 Agility potential that can serve as an AQ test. The questions that follow (see Figure 4) are but a small sample of those that are suggested by these lessons learned as well as other lessons learned and reported on in the NATO Research Group SAS-085's Final Report on C2 Agility ${ }^{11}$.

\section{Questions: C2 Agility Potential}

- What is the most network-enabled approach that can be adopted?

- How many different approaches to $\mathrm{C} 2$ can be adopted?

- How is the approach to C2 initially determined?

- Is the appropriateness of the C2 Approach periodically assessed?

- Is the way C2 is currently being approached monitored?

- Are there processes in place to ensure that the $\mathrm{C} 2$ approach is balanced?

- Is the state (performance) of supporting systems monitored?

- How agile are individuals, processes, and supporting systems?

Figure 4: Question re: C2 Agility Potential

A Model of Potential System Agility

C2 Agility depends to a significant extent on the agility of the systems that support C2. More agile systems are less likely to impose constraints on an entity's choice of C2 Approach. Fortunately, system agility is of increasing interest to the system engineering community. There is an interest in improving both the agility of the processes that design and develop systems (agile acquisition) as well as the agility of the systems themselves (agile systems). Both are important to the C2 community as more agile systems will improve C2 Approach Agility and more agile acquisition will enable us to field systems with needed capabilities more rapidly and efficiently.

\footnotetext{
${ }^{11}$ This report and its appendices can be found at http://dodccrp.org/html4/sas-085.html
} 
Over the years, systems engineers have discovered a host of techniques (modularization, table driven logic, etc.) and associated standards have been employed to reduce the time and costs associated with responding to new requirements, one aspect of system responsiveness. Means have also been found to handle spikes in workload (e.g.; spawning of new processes and prioritized queues) that eliminate or mitigate the adverse consequences of increases in workload. System resilience is an area that has received considerable attention. As a result ways to deal with outages, delays, and loss of functionality are being designed into systems.

A number of systems engineering principles are thought to, if followed, produce more agile systems. These principles are related to reusability, re-configurability, and scalability ${ }^{12}$. These means of enabling agility, as well as others that may be identified, can provide the basis for the development of agility 'markers', variables that measure the degree to which a means has been achieved. These markers can serve as indicants of potential agility and can be integrated into an agility value proposition. Systematic experimentation is needed to validate these markers and refine our understanding of the agility value chain. The aim of a model of potential agility is to integrate all of these means and markers into a value proposition; one that enables 'designers' of organizations and systems (e.g.; commanders, managers, engineers) to better understand how they can enhance an entity's potential agility and to do so efficiently.

Summary

This paper has asserted that agility is an entity capability that can no longer be relegated to a “nice to have” requirement. It has explained that an entity's potential agility cannot adequately be ascertained by employing only scenario-based approaches to the development of requirements or for testing the degree to which an entity is agile. It concludes that a conceptual model of potential agility, one based upon an entity's characteristics and behaviors under stress is needed and it suggests an approach to the development of such a model.

\footnotetext{
${ }^{12}$ Dove and LaBarge (2014) Fundamentals of Agile Systems Engineering INCOSE
} 


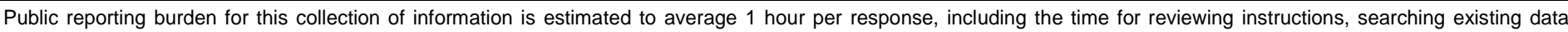

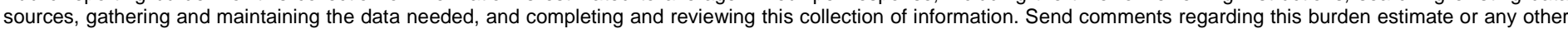

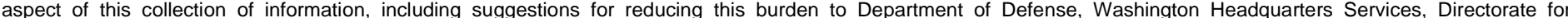

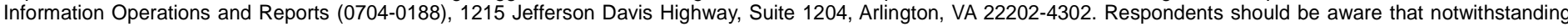

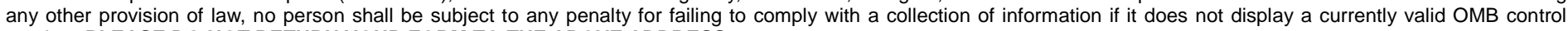
number. PLEASE DO NOT RETURN YOUR FORM TO THE ABOVE ADDRESS.

1. REPORT DATE (DD-MM-YY) April 2014

\section{TITLE AND SUBTITLE}

Agility Quotient (AQ)

6. AUTHOR(S)

David S. Alberts

\section{REPORT TYPE}

Final
3. DATES COVERED (From - To)

5a. CONTRACT NO.

DASW01 04 C 0003

5b. GRANT NO.

5c. PROGRAM ELEMENT NO(S).

5d. PROJECT NO.

5e. TASK NO.

AA-6-3630

5f. WORK UNIT NO.

8. PERFORMING ORGANIZATION REPORT NO.

IDA NS Document D-5174

Institute for Defense Analyse

4850 Mark Center Drive

Alexandria, VA 22311-1882

10. SPONSOR'S I MONITOR'S ACRONYM(S)

9. SPONSORING I MONITORING AGENCY NAME(S) AND ADDRESS(ES)

Office of the Deputy Assistant Secretary of Defense (C3 \& Cyber)

Office of the Under Secretary of the Under Secretary of Defense for

Acquisition, Technology, \& Logistics

11. SPONSOR'S I MONITOR'S REPORT NO(S).

Washington, DC 20301

12. DISTRIBUTION / AVAILABILITY STATEMENT

Approved for public release; distribution is unlimited.

13. SUPPLEMENTARY NOTES

\section{ABSTRACT}

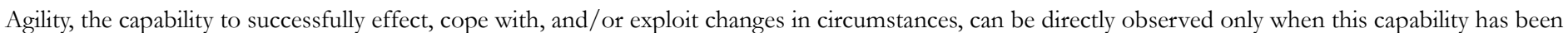

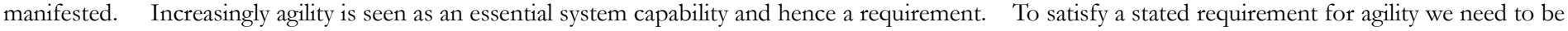

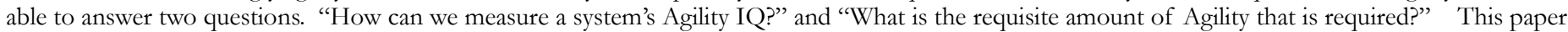
suggests a way forward and illustrates it, in the context of C2 systems.

15. SUBJECT TERMS

Systems Agility, C2, Command and Control, C2 Agility, Potential Agility

16. SECURITY CLASSIFICATION OF:

a. REPORT

U b. ABSTRACT

U
17. LIMITATION OF ABSTRACT

UU
18. NO. OF PAGES

14 19a. NAME OF RESPONSIBLE PERSON

Davis S. Alberts

19b. TELEPHONE NUMBER (Include Area Code)

(703) 845-2411 




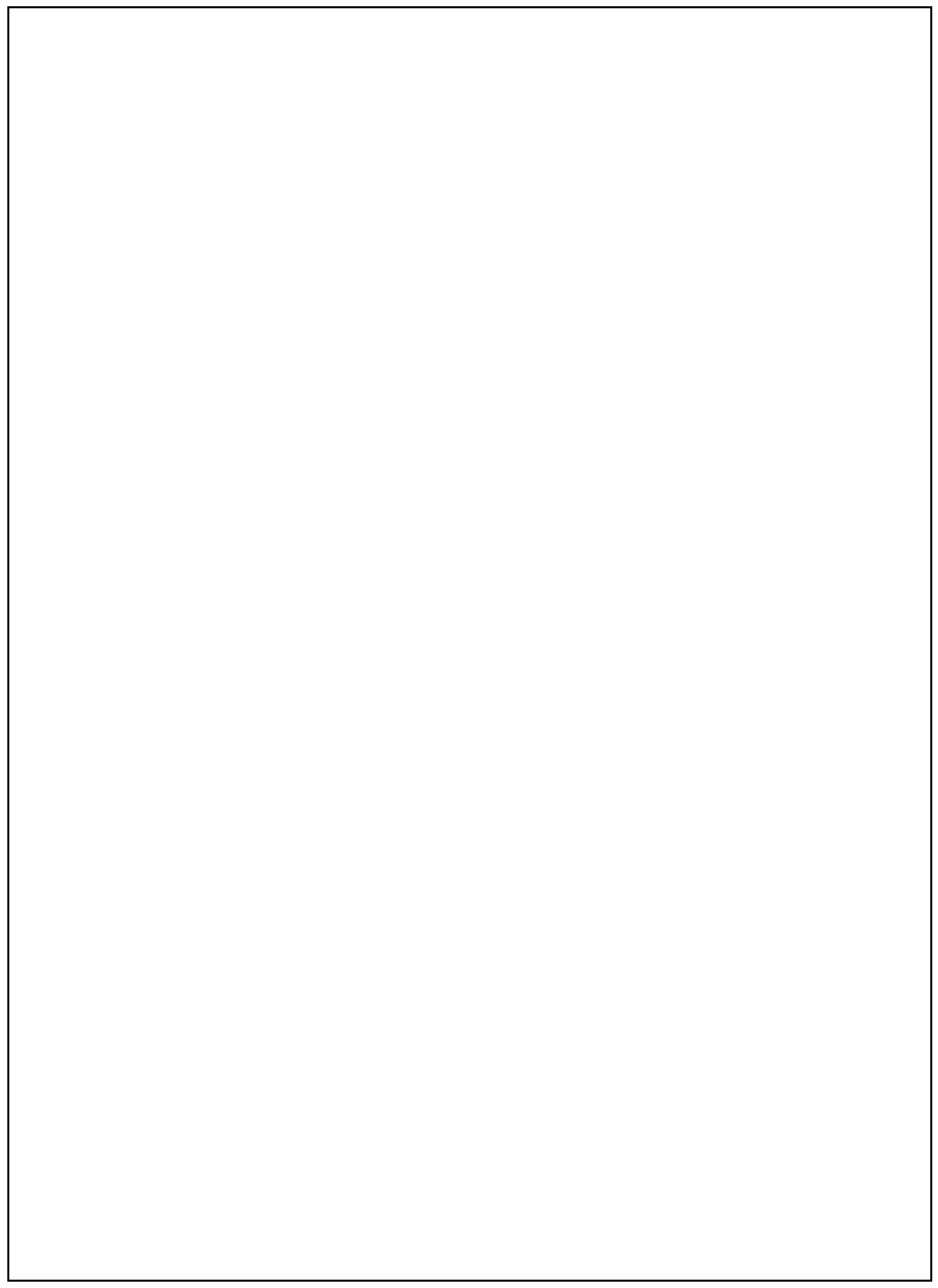

